\title{
ANALISIS DE UN GRUPO MULTIFAMILIAR DESDE LA PERSPECTIVA SOCIODRAMÁTICA
}

\author{
MARÍA JÁÑEZ ÁLVAREZ ${ }^{1}$ \\ CSM, Puente de Vallecas, Madrid
}

Iniciamos este trabajo con la definición de dos dispositivos terapéutico, el grupo multifamiliar (GMF) y grupo sociodramático (SD), para continuar con su comparación. Tras analizar sus similitudes y diferencias, descubrimos su potencial complementario para facilitar una experiencia emocional correctiva donde identificar las «tramas enloquecedoras» e iniciar otras más sanas. Así también, vemos que la urdimbre de los distintos contextos implicados hace posible la claridad y accesibilidad al cambio, siendo tan importantes sus límites, como su presencia.

Palabras clave: grupo multifamiliar, psicodrama, sociodrama, integración técnica.

We began this work with the definition of two therapeutic devices, the multifamily group (GMF) and the socio-dramatic group (SD), to continue with their comparison. After analyzing their similarities and differences, we discover their complementary potential to facilitate a corrective emotional experience where to identify the «maddening plots» and to initiate healthier ones. We also see that the warp of the different contexts involved makes clarity and accessibility to change possible, their limits being as important as their presence.

Key Words: multifamily group, psychodrama, sociodrama, technical integration.

English Title: ANALYSIS OF A MULTIFAMILY GROUP FROM A SOCIODRAMATIC PERSPECTIVE

\section{Cita bibliográfica / Reference citation:}

Jáñez Álvarez, M. (2021). Análisis de un grupo multifamiliar desde la perspectiva sociodramática. Clínica e Investigación Relacional, 15 (2): 419-435. [ISSN 1988-2939] [Recuperado de www.ceir.info ] DOI: $10.21110 / 19882939.2021 .150208$

\footnotetext{
${ }^{1}$ Psicóloga clínica del Centro de Salud Mental Puente de Vallecas (Madrid). DIRECCIÓN: María Jáñez Álvarez; C/Ramón Calabuig, 58, Bajo 2; 28053 Madrid; 620432288; maria.janez@salud.madrid.org
} 
«...lo que los labios se callan, lo dice la punta de los dedos...»

Bour (1977, p. 313).

\section{JUSTIFICACIÓN DEL INTERÉS}

Cuando conocí la Terapia Multifamiliar estaba estudiando psicodrama. La experiencia me fascinó, aunque todo lo que oía pasaba por el traductor del interés de ese momento. Hace un tiempo, asistí a unas Jornadas en Madrid presididas por J. García Badaracco (2007). En una de las conferencias visionamos un vídeo y luego hubo un coloquio. No podía parar de imaginar posibles escenas psicodramáticas auxiliando las intervenciones de los protagonistas de la escena real. Desde entonces no he parado de estudiar los componentes terapéuticos comunes entre la Terapia Multifamiliar y el Psicodrama.

Esta idea se reavivó durante los años en que participé en los Grupos Grandes del Máster de Grupoanálisis de Fundación OMIE (de 2011 a 2014) que, sin estar integrados por Grupos de Familias, parten de Grupos Pequeños (como familias) de miembros muy distintos. Así también a partir del momento en que empecé a conducir Grupos Multifamiliares en 2017.

Dado mi entrenamiento en Psicodrama, y después de profundizar en la comprensión de los Grupos Grandes (GG), me planteo un ejercicio de mirar a los Grupos Multifamiliares (GMF) desde la perspectiva del Sociodrama (SD). Espero que esto sirva para sumar a las herramientas que me da la teoría y experiencia en Grupoanálisis, las que me dio, y me sigue dando, el Psicodrama.

\section{PRESENTACION DE LOS DISPOSITIVOS: GRUPO MULTIFAMILIAR Y SOCIODRAMA}

\section{GRUPO MULTIFAMILIAR}

El Grupo Multifamiliar (GMF) es una terapia de grupo pensada originalmente para pacientes con Trastorno Mental Grave institucionalizados. Está inspirada en el modelo psicoanalítico y la terapia familiar. Trata de analizar las relaciones vinculares que suceden en el grupo, abriendo espacios para nuevas experiencias a partir de las cuales poder desentramar relaciones de interdependencia patógena, resolver duelos patológicos y fomentar recursos yoicos.

Esta intervención se caracteriza técnicamente por: la indicación de la no interpretación y privilegio del uso de clarificación y confrontación, la actitud activa del conductor, y la indicación de no actuación fuera del encuadre; en su lugar se aprovechará la regresión del Grupo Grande para facilitar el acceso a contenidos reprimidos y, sin dejar de analizar la relación 
con los conductores, privilegiar la relación entre los asistentes para limitar la dependencia regresiva.

El creador de los GMF, J. García Badaracco, inició sus reuniones multifamiliares en 1960 en el salón de estar del Hospital Borda de Buenos Aires, con 40 internos considerados «crónicos». En este espacio se reunían los profesionales con todos los pacientes al mismo tiempo, incluyendo a sus familias; esto transfiguraba dicha sala en una Comunidad Terapéutica. Los asistentes planteaban sus dificultades relacionales. Estas conversaciones tenían un flujo libre y respetaban los turnos de los participantes. Empezaron siendo reuniones de pacientes y profesionales, pero acabaron incluyendo a los familiares porque descubrieron que la presencia de muchas familias junto al equipo terapéutico constituía un campo relacional amplio, que permitía desplegar, revivir y, sobre todo, pensar las experiencias emocionales que estaban en la base de las interdependencias patógenas. Badaracco explica esta idea, en base a la experimentación con el encuadre de GMF de los primeros años, del siguiente modo:

«Las expresiones de la locura eran mucho más contenidas en el grupo grande que en un grupo chico o en individual. Nos dábamos cuenta de que en el grupo grande disminuía el temor a hacer daño [...] y la violencia podía expresarse más abiertamente [...]. Hasta el punto de que, cuando una familia se atreve a abordar su problemática, con toda la carga de violencia reprimida [...], esto se constituye en una experiencia emocional que por identificación viven todos los demás, cada uno con sus posibilidades» (Badaracco, 1995, pp. 9-10).

A partir de esta práctica, y en base a toda la formación e influencias adquiridas en los años previos, Badaracco planteó que la causa de la dificultad de adecuación del encuadre clásico psicoanalítico a las peculiaridades del paciente definido como grave, venía determinada por la indiferencia con que estos se presentan ante otros (Badaracco, 1995). Esta desconexión aparente la entendía como una «alienación», construcción mental que intentaba neutralizar el sufrimiento psíquico provocado por las interdependencias patógenas, que se activaban como transferencias psicóticas masivas (Badaracco, 1998, 2000) ante el contacto con otros, especialmente con sus familiares. A partir de ellas, el paciente construye «personajes» detrás de los que se esconde. Siguiendo esta idea, también descubre que los pacientes participantes (en su mayoría psicóticos) pueden compartir y replantear estos personajes.

Vamos a representar una escena de un $\mathrm{GMF}^{1}$ para ilustrar este encuadre e identificar esta idea de «personaje» en el que se protege el psicótico:

Todos estaban reunidos desde hacía un cuarto de hora. Eran unos cuarenta. Tres de ellos eran profesionales sanitarios y el resto representaban a unas diez familias. No era la primera sesión de grupo. Todos habían sido invitados por sus profesionales referentes del Centro de Salud Mental al que acudía alguno de los miembros de la familia participante. La asistencia era voluntaria y el 
turno de intervención libre, en general los participantes compartían preocupaciones y dificultades cotidianas del funcionamiento familiar. Después de una breve presentación, llegó el silencio y la invitación a participar.

Darío se mostraba muy tenso, retorcido en la silla y con los puños apretados, se veía claramente afectado tras la presentación de su madre que era la primera vez que asistía a este grupo. Ella había hablado como si él no estuviera presente.

Tal cuál planteó Badaracco, el objetivo del GMF consistiría en desmontar los personajes («al esquizofrénico», «al chico peligroso», «al retorcido», etc.) en los que están atrapados los pacientes «graves» debido a la trama familiar. Primero, revelando las interdependencias patógenas que han intervenido en su construcción (relación paterna, fraterna, etc.), para después deconstruirlas y lograr la desidentificación que permita la emergencia del verdadero sí-mismo del paciente. Para que esto sea posible, es necesario que durante la sesión del GMF se de un intercambio comprometido de experiencias concretas, lo que permitirá visualizar las semejanzas de unos con otros y devolverá la posibilidad de pensarse, lográndose así la flexibilización de los roles fijos. Para este fin de identificación y modificación de patrones, la teoría de roles (Filgueira, 2010, p. 240-243) podría ser un elemento teórico de ayuda. Tomando esta teoría y analizando los primeros patrones que se dibujan en la escena de la viñeta clínica podemos captar un racimo de roles con una estructura particular; si tomamos el recurso de la escultura para definir la configuración de estos roles (complementarios), podríamos describirla con el título de: «alguien retuerce o es retorcido por otro alguien». A partir de esa representación podemos captar la experiencia colectiva de un momento y profundizar en la trama familiar puesta en escena.

\section{UNA MIRADA PSICODRAMÁTICA}

El modo de intervención que se acaba de plantear evoca en cierta manera algunos de los planteamientos del modelo psicodramático. Cabe recordar, que el Psicodrama es un método de psicoterapia en el cual los pacientes representan² los acontecimientos (o fantasías) relevantes de su vida en vez de simplemente hablar sobre ellos (Blatner \& Blatner, 1988, p.1). Así, además de lograr una comprensión más profunda de sus acciones, pensamientos, emociones y de sus consecuencias, la persona conseguirá generar espontáneamente una respuesta distinta a la situación problemática que ha planteado para la dramatización; con un nuevo desempeño del rol representado, más adaptativo y generalizable a su vida.

Un ejemplo de esto fue el primer trabajo que Moreno realizó con Bárbara y Jorge, colaboradores de su Teatro de la Espontaneidad (1922-1925), relativo a sus propios roles de 
relación de pareja. En este trabajo se aprecia muy bien cómo en la medida en que Moreno fue ofreciendo a Bárbara un escenario y dándole papeles de esposa vengativa y novia rencorosa, las escenas jugadas en casa con su marido empezaron a subir a la escena psicodramática, en conexión con los episodios de la infancia y con la posterior reflexión. De este modo, fueron bajando las escenas trabajadas a su vida, y aumentando su comprensión sobre la misma, haciéndose más satisfactoria la relación entre ella y su marido (Moreno, 1978).

Antes de tomar las herramientas del psicodrama para mirar el grupo, cabe recordar que el origen del psicodrama se remonta a 1921, aunque su difusión internacional no tuvo lugar hasta el año 1949. Inicialmente fue pensada como una psicoterapia grupal que fue abarcando con el tiempo otros encuadres individuales, familiares, etc. Muchos de sus conceptos teóricos y prácticos se han ido integrando con otras corrientes como el Psicoanálisis, la Gestalt, la Terapia Sistémica y otras Terapias Corporales.

En general, el psicodrama es una terapia de las relaciones que considera que no se puede percibir a un sujeto de manera aislada a su contexto (Moreno, 1978). Según el foco de la conducción del grupo, podemos hablar de varios enfoques posibles: el psicodramático, el sociodramático y el axiodrama (Álvarez-Valcarce, 1997). El más próximo o compatible con el GMF es el sociodramático (SD) porque estudia y explora los elementos sociales y colectivos de los roles y su dinámica conflictiva en la escenificación terapéutica.

"Los objetivos para el trabajo sociodramático apuntan en general hacia el desarrollo y la mejoría cualitativa de las relaciones y de las pautas de conducta o roles asociados con ellos, tanto en el plano individual como en el de la sociedad y la cultura; en contraste con el Psicodrama que explora más las representaciones internas de estas relaciones» (Filgueira, 2010, pp. 275-6).

\section{PLANTEAMIENTO DE ESTA MIRADA SOCIODRAMÁTICA (SD) DE UN GRUPO MULTIFAMILIAR (GMF)}

El modo con que Badaracco define el objetivo de estos GMF evoca un escenario psicodramático con enfoque sociodramático, donde desentrañar algo de la trama familiar que atrapa al paciente mental grave. Es decir, aunque el encuadre multifamiliar es un escenario particular distinto al sociodramático (SD), tiene resonancias con él que subyacen al concepto de «virtualidad sana» y que analizaremos en este apartado.

En relación con esta mirada $\mathrm{SD}$, sintónica con el GMF, cabe destacar que mientras en la terapia familiar el elemento de análisis es el paciente, en el GMF es la familia. De modo que el paciente es meta analizado en grupo (Caparrós, 2013, pp.163-208). «El estudio de las relaciones interpersonales permite profundizar en la naturaleza de las dinámicas intrapsíquicas. Esto es 
más evidente en los pacientes psicóticos o con neurosis graves en los que la actividad psíquica, que tiene poca autonomía, está, por así decir, siempre muy referida a otro y se despliega en el campo de la relación con los demás; siempre relacionado con un Superyó parental» (Badaracco, 1990, pp.149-150). «El hecho de incluir a las familias permite revivir los conflictos primitivos y elaborarlos, también con los objetos reales con quienes se estructuraron originalmente; discriminando entre objetos internos y externos» (Badaracco, 1990, p.170).

Mascaró (2012) analiza los efectos de esta configuración multifamiliar y concluye que funciona como disparador para la «desalienación» de la trama familiar patógena; explica esta cualidad relacionándola con que en estos grupos «la transferencia se reparte entre sus miembros, reduciéndose la carga emocional» (p. 37). Por otra parte, el contacto con otras figuras identificativas descubre aspectos de la vida que no hemos vivido y podrían estar a nuestro alcance. Así, a través de la convivencia, se abren nuevos espacios transicionales, tanto reales como virtuales. Según esto, y una vez logrado un clima de confianza, añadiremos a los contextos manifiestos del GMF —el grupal y social_-, el potencial de un contexto virtual. Este tercer posible contexto evoca de nuevo el contexto psicodramático (PD), en este caso como un espacio mental tanto del que mira como del que es mirado. Podemos imaginarlo como un escenario en el que el grupo aparece como una escultura en movimiento, donde los actores se presentan como «personajes» en relación, en el que pueden tomar distancia, mirarse y pensarse (McDougal, 1982; Pirandello, 2001; García-Bustamante, 2012). La presencia de este tercer contexto dependerá de que quien mira pueda incluirlo e incorporarlo como un lugar de lo posible, sino quedará silente tras la interacción manifiesta.

Si continuamos con el ejemplo del GMF que apenas acaba de empezar, podemos explorar algunas de sus posibilidades para iluminar una escena narrada, según la perspectiva sociodramática, en consonancia con la línea que sugiere Badaracco. A continuación, transcribimos la escena descrita previamente en la voz del director (psicodramático) combinando su función de analista, conductor y productor dramático:

La trama está puesta en escena. Los protagonistas son una madre y un hijo.

Parece que la posición forzada de Darío es una reacción a los comentarios de su madre que parece no verle.

La lectura de formas corporales invita a iluminar el campo de tensión, trabajando sobre una «imagen real corporal» que está teniendo lugar. Si no se facilita la expresión de esta forma tensa, tenderá a ser actuada o a aparecer como confusión. Pararse a pensar sobre ello, llevará a la reflexión del grupo y a que la emoción pueda ser metabolizada. 
Siguiendo el mensaje de esta viñeta, cabe analizar con detalle otra de las características implícitas más reseñables de estos GMFs, próximas al SD: la peculiaridad de que los familiares estén físicamente, lo que implica una gran viveza de los vínculos y de las situaciones evocadas 3 . Esto permite revisar los roles en vivo (socioterapéuticamente) y contrastar sus resonancias subjetivas, o viceversa; añadiendo a esto, la potencia de la multiplicación dramática de otras familias (representantes de lo social). Es decir, los miembros de la familia erigidos como protagonistas de la escena, repiten una interacción (ponen en acción, más que relatan) en la que están atrapados, sin posibilidad de una representación subjetiva cual neuróticos. La «mente ampliada» del grupo puede ayudar a tomar conciencia de esta re-presentación primitiva, lejana al pensamiento, más propia de interacciones psicóticas. Esta virtualidad grupal facilitará cierta distancia con el personaje re-presentado (implícito en los «roles fijos») (Badaracco, 2012, p.30) $y$, en el mejor de los casos, el replanteamiento de su sentido.

Además del esclarecimiento grupal sobre el sentido de las acciones compartidas, la obra El grupo multifamiliar: un espacio sociomental (Ayerra y López-Atienza, 2003) apunta hacia el posible empleo del GMF para profundizar en la escena vivida del aquí y ahora, relacionada con la del allí y entonces (más en una línea psicodramática). Esto implica dar un paso más allá de lo representado para desentrañar el rol en el que el paciente se siente atrapado.

«En el escenario del GMF intentamos conseguir un espacio psicodramático donde expresar ese complejo mundo interno y re-presentar las experiencias donde el paciente designado ha quedado atrapado como un personaje sin papel elegido. Este escenario permite una vuelta al pasado para recuperar el sentido y también una puerta al presente donde reconocerse como persona en un contexto social complejo, con un futuro por escribir» (Ayerra y López-Atienza, 2003, p. 8).

Esta cita condensa muy bien el lugar de encuentro posible entre GMF y PD. Si reflexionamos sobre este lugar común, en el fondo el PD no añade nada nuevo a la acción del GMF, pero es un instrumento «intra-intermediario» (Rojas-Bermúdez, 1972, 1997) del conductor que facilita la mentalización e instrumenta un «espacio virtual» (Badaracco 2012, p. 29), donde la espontaneidad y la creación son posibles. Es decir, la posibilidad de que el conductor del GMF mire la escena con «ojos de conductor psicodramático», incluye este tercer posible contexto como espacio intermediario donde representar el contexto grupal y profundizar sobre lo no dicho.

Si continuamos con el ejemplo (del grupo, y en concreto con esa escena en que una madre y un hijo se desencuentran ante la mirada de todos) y analizamos la función del director que capta una forma tensa y lo explicita para el grupo, descubrimos que no es más que un lector de lo que pasa, que, al dirigir el foco de atención a una forma corporal y «reflejarlo» (Dozza, 2014, p.181), permite formas nuevas. Es un facilitador, que acompaña, poniendo cuerpo y mente a lo que 
sucede, a veces en silencio y otras poniendo voz a lo que ve, con preguntas no directivas que buscan que la tensión no sea ni demasiado alta, ni demasiado baja. Vemos que no se diferencia mucho de las posibles intervenciones de un conductor de GMF, pero la dimensión escénica ofrece una paleta amplia de lentes con las que mirar la escena, lo que puede aumentar los matices percibidos4.

De este modo, si la acción no llegara a ser una re-presentación para el protagonista mismo de la escena, aún podría ser una representación para otros, ya que las resonancias 5 de estos dramas trascienden lo personal. Es precisamente ahí donde se sitúa la aportación de este trabajo: en que lo que el conductor con este «mirar sociodramático» llega a intuir sobre ese allíy entonces, pueda hacerse manifiesto en el escenario grupal del aquí y ahora. Es decir, no tratamos de traducir la dinámica grupal en términos sociodramáticos, sino de mostrar cómo las herramientas sociodramáticas amplifican la capacidad de análisis del mismo grupo. Además del reflejo del conductor de estas resonancias, cabe recordar algunas otras herramientas: por ejemplo, ante las proyecciones y distorsiones de la comunicación, producidas por los conflictos individuales, el Psicodrama ofrece el esclarecimiento a través de sus técnicas específicas de producir reacciones y consecuencias en otros. Algunas de estas técnicas tienen una clara conexión con la teoría de las estrategias defensivas o reparativas del Psicoanálisis Clásico. Una muestra de ellas sería dirigir la mirada al grupo en sí mismo como espejo de estas distorsiones, lo que contribuye a la identificación y su resolución. Siendo este un elemento común con el GMF. Así también, la clarificación para la elaboración empleada en GMF es una técnica próxima a las psicodramáticas, como el soliloquio, doble e intercambio de roles; la confrontación y el resumen son técnicas más cercanas a la interpolación de resistencia y al espejo, este más próximo al cuerpo y a las dinámicas de acción.

Sigamos ahondando en las siguientes escenas:

Darío sigue tenso, y pronto esto se representa en otros participantes. La madre de Marito toma la palabra $y$, en lugar de expresar su malestar, pide instrucciones a la conductora para tratar a su hijo. Es ahora Marito quien se violenta y le pide a su madre que se calle.

Marito funciona para Darío como un espejo, imagen corporal del otro, y a la vez un doble que dice lo latente que está en el cuerpo de Darío.

Marito parece que baja un poco los hombros, y dice susurrando:

_ ¿Logrará que se calle? (lo que sería un soliloquio, mensaje que evoca lo silente).

La conductora le pregunta a Marito por eso que escuchó en su madre que no puede soportar, luego le pide- cual intercambio de roles - que se pregunte si su madre entenderá esto que plantea de este modo: expresa. Este material es para uso científico y profesional exclusivamente y puede contener información clínica sensible. Los editores no se responsabilizan de los contenidos de los autores. Dirigir las consultas sobre derechos y autorizaciones a ceir@psicoterapiarelacional.es 
— ¿Qué es lo que dijo tu madre? ¿Qué es lo que entendiste?

- Le preguntó al doctor sobre cómo debe tratarme. ¿No sabe cómo tratarme siendo su hijo? ¿Acaso soy un monstruo? ¿Me ves como un monstruo, mamá?

El silencio inundó la escena. Todos estaban mudos.

—Es que no sé cómo hacerlo, hijo-dijo la madre llorando-.

Vemos cómo la mirada del conductor, enriquecida por la formación en Psicodrama, permite, cual doble, focalizar la atención sobre lo corporal, lo latente, lo no audible presente en la escena y profundizar en sus observaciones de las formas naturales (entendidas como elementos corporales, preverbales, paraverbales) y formas sociales (componentes verbales, que suelen recoger contenidos más elaborados). De este modo, y como venimos planteando en las viñetas clínicas, a partir de poner foco en una forma corporal, como es la figura retorcida de Darío, podemos indagar sobre la dinámica que le envuelve. Empleando diferentes técnicas identificamos una matriz materna asfixiante, que se dispara ante las conductas en las que el hijo es presentado como objeto. Cuando el conductor capta esta dimensión corporal y lo comparte con el grupo, la técnica del doble se multiplica dentro de la escena grupal y Marito la retoma, dando lugar a una multiplicación dramática. Estas técnicas son específicas del Psicodrama pero no parecen elementos extraños en la expresión del GMF, donde además de palabras, hay tonalidades y un manejo del cuerpo particular; así como la sucesión de intervenciones que ayudan a aclarar la escena central. Y es que, la configuración del GMF, convoca imaginariamente el contexto dramático, que consta de su propio tiempo (etapa de dramatización) y espacio (escenario), y en el cual los contenidos del contexto social, que circulan en el contexto grupal, se actualizan en roles y escenas.

Revisadas todas estas posibilidades técnicas, volvemos a destacar una de las cualidades de los GMF: La presencia en vivo de grupos familiares naturales hace muy presente esta dimensión corporal y facilita al protagonista la activación de experiencias «como si», trayendo al aquíy ahora el "cómo es», en contraste con sus fantasías. Este ingrediente común al GMF y SD, permite superar las limitaciones de la terapia verbal como el psicoanálisis clásico (Ayerra y López-Atienza, 2003, p. 121). La participación comprometida emocional de ciertos participantes, al producirse y tener lugar en el contexto multitudinario del GMF constituido por familiares propios, por otros pacientes y otros familiares, y también personas que intervienen no como familiares, da lugar a diálogos que permiten incluirte (Badaracco, 1995, p. 10) y pensarte, sin quedarte en frases hechas (o «conservas culturales»). De este modo, el trabajo con las instantáneas reconocidas en la escena grupal de formas y espacios corporales es captada por el conductor y el resto del grupo. La simple explicitación/reflejo de estas formas ayuda a reflexionar juntos sobre su significado (Sunyer, 2008, p. 250). En este caso, el conductor 
no llega a insinuar nada, las instantáneas compartidas son las que traen Darío, Marito y sus madres. Pasamos a continuación a describir distintas formas de reflejar estas escenas ${ }^{6}$.

Desde un foco psicodramático, el conductor podría pedir a alguno de los protagonistas la descripción detallada de lo que pasa y su relación con otras situaciones pasadas de coordenadas espaciales concretas, evocadoras de un afecto. Pero, en esta perspectiva grupal o sociodramática, no se trata de profundizar en la historia del protagonista, si no tomar las formas del protagonista como representante del grupo, como «una imagen en plural» (Elías, 1988, p.144), aunque cada uno de los miembros parta de una escena personal que tiene que ver con su historia y sus conflictos pendientes. Estos conflictos personales serán explícitos solo en la medida en que funcionen como emergentes para el grupo. Si no, se iluminarán como nuevas representaciones internas apenas audibles, donde también está incluido el conductor. Si, por el contrario, la lectura de formas y la identificación de los emergentes son adecuadas, el protagonista servirá como mediador para que todos puedan identificarse y así pensarse; ya que «la temática que surge en una familia despierta cosas similares en otras y esta espiral va enriqueciendo los temas y a su vez estimula la comunicación» (Badaracco, 1987, p.208). Esta disposición permite que se vaya creando una escena nueva en la que confluyen posiciones dialécticas, pasando de lo real a lo simbólico7. En esta línea, cabe recordar al conductor la necesidad de estar alerta a la tendencia de los Grupos Grandes de usar un lenguaje metafórico (relacionado con idealizaciones y duelos patológicos). Habrá de cuidarse y procurar salir del discurso abstracto para centrarnos en acontecimientos más próximos, pero con un escenario plural y amplio, que pueda cuestionar «lo incuestionado» hasta ahora. Recojamos la escena de Darío de nuevo y démosle otro posible giro, como ejemplo de este centrarnos en algo concreto, abriéndolo a un escenario plural:

Al igual que algunas escenas de los participantes se quedan silenciadas cuando no conectan con los emergentes grupales, así también muchas de las ideas que el conductor va captando, en especial cuando el discurso libre grupal va trayendo lo nuclear. Sin embargo, si la sesión avanza y ninguno de los participantes hubiera retomado escenas nucleares, como la del retorcimiento de Darío, hubiera cabido que el conductor aludiera a ellas directamente, por ejemplo, apelando a Darío o a su madre para aclarar lo que está pasando.

De este modo, si Darío no hubiera podido llegar a conectar su cuerpo con la voz, a través de Marito, el conductor podría haber mantenido el foco en esa matriz madre-hijo e invitado a la madre a tomar conciencia de su discurso y su impacto (a modo de cambio de roles); reflejando sobre todo que preguntar a un tercero qué hacer con un sujeto presente puede resultar muy embarazoso, en especial si al referirse a él se dice que es alguien «que escucha sin entender y al que no hay modo 
de enderezar». Este simple espejo o doble, si hubiera sido expresado en primera persona, relajaría ${ }^{8}$ a Darío. $Y$ a otros, como Marito, que empezaban a retorcerse también.

Avanzando en esta viñeta, nos encontraremos con una exposición de cómo «las técnicas sociodramáticas permiten objetivar constantemente la diferencia entre lo que el grupo dice que ve en la realidad y lo que realmente ve» (Álvarez Valcarce, 1995a, 2011). Pudiendo unir la acción automática con los roles implicados y pasar de los roles repetidos a los elegidos, adecuados a cada situación. Tomamos la continuación de la conversación con la madre de Darío para ejemplificar esta idea:

Imaginemos que, en respuesta a la reflexión del conductor, la madre de Darío insiste en el mimo con el que trata a su hijo, lo que está en clara contradicción con la desconsideración que se observa en su rictus facial y en su posición corporal, distante y con orientación contraria al cuerpo de Darío. Además, se expresa como si ignorara su presencia.

Esta escena explicita una discrepancia; poder manifestarlo podría ayudar a que tomara conciencia, profundizara en su sentido y mejorara futuras comunicaciones. De este modo, se evidenciaría lo imaginario que esconde la acción y lo engañoso del discurso aprendido.

El hecho de trabajar en Grupo Grande, permite conocer la visión de cada uno de los presentes (por ejemplo, sobre esta discrepancia en la comunicación), y, partiendo de un objeto común, conocer visiones alternativas al respecto, lo que es en sí mismo una buena herramienta de contención. Como plantea Badaracco (2006) el mismo grupo es una especie de auditoría de lo que pasa (2006, p. 8). Al descubrir lo parcial de una mirada sobre el objeto, y al unirlo a lo parcial del otro, se revela una realidad compleja más próxima al sujeto/ y a nosotros. De este modo las presencias enloquecedoras activadas en los participantes se vuelven más comprensibles al actuarse en interdependencia con la matriz familiar de origen, desde el lugar del padre o del hijo, del objeto o sujeto, desde la víctima o su agresor, y sobre el yo sobreadaptado o superyó sádico. Este juego de contrarios que descubre la acción de roles y contra-roles, más que identificar a un enfermo, evidencia el funcionamiento de una matriz familiar sufriente y generadora de dolor transgeneracional que invita a la empatía y a practicar nuevas interdependencias más sanas (Badaracco, 2006, p. 10). Así, por ejemplo, tras la expresión de uno de los protagonistas del daño infringido por su familia, aparece la expresión de disculpas y de sufrimiento de una madre con toda una trama familiar no compartida hasta el momento. Y así sucesivamente, como una mecha que va poniendo luz en cada una de las tramas familiares más próximas al protagonista 9 .

Si añadimos a este espacio plural de multirrelaciones, el potencial de los GMF para intervenir sobre las relaciones que se están dando en el aquí y ahora (Ayerra, 1995, p. 1; Ayerra y LópezAtienza, 2003, p. 4), como la de Darío y su madre, descubrimos que el escenario GMF potencia 
el lugar del tercero. Entendiendo por tercero a «ese agente que rescata a los miembros de la simbiosis» y permite que la función terapéutica deje de ser persecutoria, como en las terapias unifamiliares, y pueda actuar como mediador. Una muestra de esto es el hecho de que una madre pueda dirigirse a la de Marito y expresar experiencias similares de un modo más accesible. En este contexto, las situaciones vinculares pueden sufrir modificaciones in situ, en la medida en que se favorezca la construcción espontánea y creativa de resoluciones alternativas. Vamos a poner varios ejemplos de estas posibilidades:

Si detecto que un supuesto protagonista como Darío tiene una dificultad marcada con su matriz materna, que parte de los momentos más tempranos, que se recrudece tras la muerte del padre, y que se dispara por algo de lo evocado en el grupo, puedo facilitar la intervención de otro paciente cuya historia sienta conectada con la trama familiar desplegada en ese momento (ya sean vínculos simbióticos o roles parentalizados a partir de una historia de duelo o maltrato, etc.). En el mejor de los casos, alguno de los participantes (ej.: su hermana) se ofrecerá para expresar comprensión y validar la emoción del protagonista, o para canalizar el malestar sentido «en propias carnes», como doble o multiplicación dramática espontánea sobre las que seguir trabajando.

A veces, estos participantes podrían no compartir dinámicas familiares, y en su lugar ser miembros de familias con otras dinámicas de funcionamiento (o en proceso de cambiarlas) que pueden aportar una mirada distinta hacia Darío o Marito, devolviendo visiones de ellos sanas y generadoras de otras posibilidades de relación. Este podría ser el caso de Emilia que se dirige a Marito diciéndole que entiende que a veces nos quedamos entrampados en relaciones difíciles pero que ella no ve en él ningún monstruo... todo lo contrario, que sus intervenciones le despiertan mucha ternura... y, dirigiéndose a la madre de Marito, le dice que la entiende y se identifica con lo que cuenta en lo que respecta a asustarse y ver monstruos fuera de sí que aumentan la tensión del ambiente. Acaba diciendo que lo único que le sirve en esos momentos es recordar que los monstruos son sus miedos, respirar hondo, buscar entender lo que pasó y me llevó de nuevo aquí, y quizás si no puedo sola, pedir ayuda.

Estas dinámicas facilitadoras de cambios relacionales precisan del cuidado del clima emocional del grupo y de la resonancia afectiva entre todos los participantes, aspecto también importante en el Psicodrama. Para ello cabe recordar que la premisa principal para intervenir ha de ser «hablar desde uno mismo», desde la propia experiencia, y compartir lo que se siente en relación a la intervención del otro, conectando con la propia historia y evitando consejos e interpretaciones (como expresa Emilia en el ejemplo anterior). El profesional debe seguir esta misma premisa de hablar desde su experiencia emocional, aunque complemente esta tarea principal con otras técnicas para analizar y facilitar la elaboración de lo que está sucediendo. En expresa. Este material es para uso científico y profesional exclusivamente y puede contener información clínica sensible. Los editores no se responsabilizan de los contenidos de los autores. Dirigir las consultas sobre derechos y autorizaciones a ceir@psicoterapiarelacional.es 
esta línea, el clima emocional de solidaridad y escucha comprometida será un elemento importante para que se gesten cambios en el modo de proyectarse e incorporar las proyecciones de otros. Estos cambios serán experimentados primero en el grupo, casi como una representación «teatral» (McDougal, 1982; Bour, 1977, p.32), para luego pasar al contexto social de cada cual.

De este modo, y volviendo al caso que venimos trabajando: el planteamiento es que si Darío, o Marito, recibieran el cariño y reconocimiento ansiado en el mismo grupo, sintiéndose respetados en el modo particular en el que hacen las cosas, su violencia (o tensión) dejaría de tener sentido (como resulta al final). En la medida en que tomen conciencia de que su agresividad viene de esa demanda básica de afecto y reconocimiento, y empiecen a buscarlo donde pueden encontrarlo, es más probable que sus síntomas remitan. Aún más, si se reequilibrara la asimetría familiar; esto es, si sus cuidadores principales llegaran a entenderlo y en lugar de responder con agresividad, empezaran a hacerlo con afecto; o por lo menos a no negar su agresividad y disculparse. Lo interesante es rescatar a los protagonistas de la compulsión a la repetición y acompañar a descubrir otros modos de relacionarse y satisfacer sus necesidades.

En este sentido y a modo de resumen, podíamos concluir que el SD coincide con los GMF en sU propósito de fomentar la salud, enfocando lo vincular que se esconde tras los roles estereotipados herederos de las matrices de identificación o vínculos primarios. Para ello es preciso el insight de lo que no nos permite ser flexibles/auténticos y a la vez un outsight, que congenia con los conceptos de acción y rematrización -psicodramática-, (y recontextualización -grupoanalítica-). El ejemplo que venimos dibujando da cuenta de ello al mostrarnos cómo en este contexto terapéutico las situaciones vinculares que tienen lugar, pueden sufrir modificaciones in situ. Si esto se logra, los miembros del grupo podrán relacionarse de una nueva manera a través de las escenas y juegos dramáticos, tanto dentro como fuera del grupo.

Conectando con el principio de la sesión, una muestra de esto es cómo después de que el conductor empleara un tiempo de reflexión compartida en torno a la forma tensa que envolvía a Darío en el grupo (dentro y fuera), el cuerpo de éste empezó a mostrarse suelto y relajado, incluso sonriente. Cabe destacar que esta posición relajada comenzó de algún modo cuando el director puso foco en esta forma y, a partir de ahí, dibujó el contorno de un escenario sociodramático imaginario, que compartió con el grupo mediante técnicas psicodramáticas. Cuando este foco se convirtió en un contenido compartido, fue repetido por otros participantes como Marito, y a partir de ahí pudo ser elaborado entre todos. Cabe recordar que éste sólo es el principio de un cambio grupal que persigue mejoras más allá de las formas corporales grupales. 
El caso planteado pretende ser un ejemplo de cómo emplear el contexto dramático (imaginario) en los GMF, para que el contexto real, sumado al contexto social de otras familias, pueda favorecer un primer paso hacia la salida de las interdependencias patógenas, ofreciendo otros modelos y fortaleciendo los «recursos yoicos». De este modo el contexto dramático se convertiría en un «espacio virtual» donde mirarse y pensarse, primero en el espejo de los otros y luego en las vivencias comunes reconocidas como propias (Badaracco, 2012, pp. 32-33).

La existencia en los átomos sociales de estereotipias y rigideces vinculares, a veces muy fuertes, hace que los miembros del grupo tengan que luchar por ser de un modo diferente en un contexto social que no es tan abierto y permisivo como el contexto grupal terapéutico. Por ello es tan importante el trabajo Multifamiliar, o la Terapia Familiar Sociodramática (ÁlvarezValcarce, 1995b), donde «lo tratado» invoca algo nuevo, todavía intangible, aunque ya manifiesto en el malestar de las escenas representadas, cuya expresión por sí misma es el principio del cambio.

Disponer de estas herramientas sociodramáticas ayuda a mejorar la disposición para identificar formas corporales y simbólicas potenciales, reforzar un clima de seguridad y, a la vez, alimentar la libre expresión emocional ajustada a la posibilidad de cambio de cada momento del grupo; permitiendo así la generación de «recursos yoicos más genuinos». Una captura afinada de estas formas naturales, complementada con las formas simbólicas potenciadas al pensar con otros, ayudará a fijar el foco sobre uno o dos protagonistas en cada sesión y re-presentar los roles en conflicto emergentes (lo que viene a ser la «trama enfermante»); y a partir de ahí reflexionar juntos sobre lo que esto implica y las alternativas para enfrentarlo. De este modo, el uso de estas técnicas activas permite potenciar la capacidad de este dispositivo GMF para rescatar al enfermo designado de su rol aislado, al comprometer al resto de la familia y auditorio en el reconocimiento de contra-roles complementarios que lo activan.

\section{CONCLUSIONES}

Por todo lo expuesto, considero que la integración de diversas técnicas permite ampliar el punto de vista de la base teórica inicial. De hecho, este es el punto de partida con el que se gestó el encuadre GMF tal como lo conocemos hoy ${ }^{10}$. En concreto, la mirada sociodramática puede ayudar en la comprensión de los fenómenos que acontecen en grupos complejos como los GMF.

El principio que dirige el modelo psicodramático, la acción (representada), no es tan discordante con las terapias verbales como a veces se plantea. En general, más allá de este principio, cuando analizamos las diferencias entre ambos dispositivos, las distancias 
disminuyen de manera notable y sus diferencias aparecen como oportunidades complementarias para la intervención.

El modo de operacionalizar esta complementariedad puede tomar formas distintas, en concreto en este trabajo tomo la opción que consiste en subordinar el dispositivo sociodramático al GMF, donde el conductor mira al grupo añadiendo un contexto psicodramático imaginario como un espacio para el análisis y la generación de hipótesis que faciliten un cambio terapéutico.

Por concluir, quiero destacar que la inclusión del sociodrama en el estudio de la dinámica grupal, sería una interesante oportunidad para el entrenamiento de futuros conductores de GMF. Cabría seguir explorando esta y otras posibles aplicaciones.

\section{AGRADECIMIENTOS}

Mi más sincera gratitud a todos aquellos que me habéis acompañado a dar forma escrita a estas ideas. Listo, respetando cierta secuencia de participación, a algunos de los que habéis contribuido de una manera más activa: a los compañeros del Máster de OMIE de Barcelona; a José María Ayerra; Pablo Álvarez-Valcarce; Equipo GMF de Getafe, sobre todo a Esther GarcíaBustamante; José Luís López-Atienza; Maribel Blajakis; Mar Soler; José Miguel Sunyer; Manu Palomo; Pilar Matamala; Silvia Álvarez-Hernández y a nuestro GMF de Puente-Vallecas. Un agradecimiento especial a Nicolás Caparrós, cuya partida me ha alentado al empujón final.

\section{REFERENCIAS}

Álvarez-Valcarce, P. (1995a). Psicodrama y sociodrama. Teoría de la técnica. Las técnicas de sociodrama. Informaciones psiquiátricas, 140, 169-192.

Álvarez-Valcarce, P. (1995b). Teoría del sociodrama familiar. Informaciones psiquiátricas, 140, 147167.

Álvarez-Valcarce, P. (1997). Axiología y axiodrama. Actas del primer congreso iberoamericano de psicodrama. Salamanca.

Álvarez-Valcarce, P. (2011). Key elements in a sociodramatic approach to groupwork. In R. Wiener et al. Sociodrama in a Changing World, pp. 91-111. USA: Lulú Ed.

Ayerra, J. M. (1995). Grupo Grande. II Congreso Nacional APAG. GETXO.

Ayerra, J. M. y López-Atienza, J. L. (2003). El grupo multifamiliar: un espacio sociomental. Avances en salud mental relacional, 2 (1). Recuperado de https://studylib.es/doc/8321327/el-grupomultifamiliar--un-espacio-sociomental. 
Badaracco, J. E. (1987). Psicoanálisis Multifamiliar. Los otros en nosotros y el descubrimiento del sí mismo. Barcelona: Paidós. Psicología Profunda.

Badaracco, J. E. (1990). Comunidad Terapéutica Psicoanalítica de Estructura Multifamiliar. Tecnipublicaciones S.A.

Badaracco, J. E. (1995). El Grupo Multifamiliar en el contexto de la psicoterapia en general. II Congreso Nacional APAG GETXO.

Badaracco, J. E. (1998). Psicoanálisis Multifamiliar para el abordaje de la patología mental grave. Psychotherapies, 18(1), 3-14.

Badaracco, J. E. (2000). Psicoanálisis Multifamiliar. Los otros en nosotros y el descubrimiento del si mismo. Buenos Aires: Paidós.

Badaracco, J. E. (2006). El psicoanálisis Multifamiliar: cómo curar desde la virtualidad sana. Revista de Psicoanálisis, LXII (4), 919-936.

Badaracco, J. E. (2012). Psicoanálisis multifamiliar. Teoría y práctica grupoanalítica, 2(1), 25-34.

Blatner, A. \& Blatner, A. (1988). Foundations of Psychodrama: History, Theory and Practice. New York: Springer.

Bour, P. (1977). El psicodrama y la vida. Madrid: Biblioteca Nueva. Campos, J.; Fidler, J. W.; Guimón, J.; Däes, R. y col. (1986). La formación en psicoterapia de grupo y psicodrama. Barcelona: Argot.

Caparrós, N. y Cruz-Roche, R. (Dirs.) (2014). El psiquismo. Un proceso hipercomplejo. Madrid: Biblioteca Nueva.

Dozza, L. (2014). Acompañamiento terapéutico y clínica de lo cotidiano. Buenos Aires: Letra Viva.

Espina-Barrio, J. A. (2001). Integración del psicodrama con otras líneas teóricas. Rev. Asoc. Esp. Neuropsiquiatría, 21 (77), 33-49.

Elías, N. (1988). Sociología fundamental. Barcelona: Gedisa.

Filgueira, M. (Coord.). (2010). Manual de Formación de la Asociación Española de psicodrama. http://www.lulu.com/product/paperback/

García-Bustamante, E. (2012). La función por hacer. Commedia da fare. Teoría y práctica grupoanalíticas, 2 (1), 137-46.

Mascaró, N. (2012). El Grupo de Psicoanálisis Multifamiliar. Teoría y práctica grupoanalítica, 2 (1), 335-44.

McDougal, J. (1982). In Theatre of the Mind: Illusion and Truth on the Psychoanalytical Stage. New York: Basic Books.

Moreno, J. L. (1978). Psicodrama. Buenos Aires: Paidós.

Pirandello, L. (2001) Seis personajes en busca de autor. Madrid: Edaf. Ediciones.

Rojas-Bermúdez, J. C. (1972). De los Objetos: transicional e intermediario. Dos formas semejantes con diferente contenido. Cuadernos de sicoterapia, 7-8, 15-35.

Rojas-Bermúdez, J. C. (1997). Teoría y técnica psicodramáticas. Barcelona: Paidós Psicología. 
Sunyer, J. M. (2008). Psicoterapia de grupo grupoanalítica. La co-construcción de un conductor de grupos. Madrid: Biblioteca Nueva.

Tolcachir, C. (2011). El viento en un violín y otros textos. Buenos Aires: Atuel.

Winnicot, D. W. (1982). Realidad y juego. Barcelona: Editorial Gedisa.

Original recibido con fecha: 30/9/2021 Revisado: 309/9/2021 Aceptado: 30/09/2021

NOTAS:

\begin{abstract}
${ }^{1}$ Se trata de una sesión, con personajes inspirados en obras de teatro de Tolcachir (2011) y en varias sesiones de GMF realizadas en el Centro de Salud Mental de Getafe en 2013 donde la autora de este artículo asistía como observadora.

${ }^{2}$ Usaremos este término como sinónimo de «(psico)dramatizar» o «actuar», entendiendo por acción un medio para el Psicodrama, siendo su propósito final la identificación de lo que nos pasa (dentro) sin que lo sepamos y el modo en que esto condiciona nuestras relaciones. Cuando logramos poner distancia entre el protagonista y su acción, y llegar a una reflexión reposada, es cuando logramos vencer lo que nos poseía y creamos una mejor disposición para resonar con nosotros mismos y con otros (Espina-Barrio, 2001). Esta actuación es representada en un escenario, con un director, un público del que emergen protagonistas que actúan el conflicto y yo-es auxiliares que intervienen modelando lo que pasa.

${ }^{3}$ Esta viveza, más que ser una diferencia, es una similitud con el SD. En el SD los integrantes son personas de grupos naturales que suelen tener un contacto frecuente, sin ser necesariamente familias.

4 En general, además del color gris, se emplea la cursiva en las viñetas clínicas para diferenciar la descripción de una escena (de la mano de un narrador o a modo de diálogo entre participantes), de la descripción o reflexión del director que expreso sin cursiva.

${ }^{5}$ Estas resonancias se expresan transferencialmente, siendo sus raíces inconscientes.

${ }^{6}$ La imagen de sí mismo tiene fundamento en el universo representacional del otro con una gama amplia de posibilidades, desde lo presimbólico a lo verbal. Según esta idea, las escenas se pueden plantear tanto en términos de especularidad visual (mirada, expresión facial, gestual, etc.), auditiva (ritmo, intensidad, tono de voz), representacional (palabra-imagen) y semántica (imágenes dibujadas y reflejadas a través del discurso) (Dozza, 2014, p.180). El manejo de estos niveles, ajustado al tipo de comunicación del sujeto, va a determinar las posibilidades terapéuticas; aspecto especialmente importante con el paciente psicótico (p.182-203).

${ }^{7}$ En esta línea, este espacio es ante todo un lugar de juego en el sentido que planteaba Winnicott (1982). Las subjetividades se entrecruzan y las experiencias de cada uno son enriquecidas por el otro.

${ }^{8}$ Esta idea evoca conceptos psicodramáticos importantes como el manejo de la iluminación y tensión de campo (Filgueira, 2010, p.376-7; Rojas-Bermúdez, 1997, p. 26). Otro ejemplo de esto aparece en la viñeta clínica de la página 11.

9 De tal manera que una persona que vivía conflictos en relación a otros- por ejemplo, por la tendencia a interpretar «intenciones malévolas» en los demás-, comienza en el Grupo a darse cuenta de que percibiendo y escuchando de una manera «más abierta» a los demás integrantes, los conflictos que vivía antes se disuelven naturalmente (Badaracco, 2006, p. 8).

${ }^{10}$ «Mi experiencia en terapia de grupo y en terapia de familia también me permitía visualizar la dimensión familiar y la dimensión grupal, inherentes a estas reuniones multifamiliares» (Badaracco, 2006, p. 2).
\end{abstract}

